\title{
"Végétal et médiations culturelles : un enjeu social, économique et culturel »
}

\author{
Compte rendu de colloque (Angers, 26-27 octobre 2004)
}

\author{
Delphine Viau \\ Animatrice scientifique, Terre des Sciences, Angers Technopole, 5 rue Fleming, 49066 Angers cedex 01, France
}

Au sein du Pôle végétal d'Angers, Terre des Sciences, centre de culture scientifique et technique, développe depuis sa création des activités éducatives, culturelles et touristiques en direction des jeunes et du grand public à partir de la science et du patrimoine végétal. Des associations, les services des Espaces verts de ville et des musées ont également mis en place des activités culturelles liées au végétal.

C'est ainsi que Terre des Sciences, avec ses partenaires scientifiques et culturels de la région ${ }^{1}$, a organisé ce premier colloque national sur le thème "Végétal et médiations culturelles ». L'objectif de cet événement, s'inscrivant tout particulièrement dans la dynamique du Pôle végétal d'Angers, était d'échanger et de faire le point sur les différentes formes existantes de médiations culturelle, éducative, patrimoniale, sociale et touristique à partir des jardins, des collections botaniques, des laboratoires, des musées et des paysages. Durant ces deux journées, le végétal a été traité comme support de médiation, mais également comme objet de médiation.

Ce colloque a réuni un public de plus de 200 personnes regroupant enseignants, animateurs (culturel, scientifique, nature, social...), responsables de services territoriaux (Espaces verts, Tourisme, Développement

\footnotetext{
Auteur correspondant : d.viau@terre-des-sciences.org

1 Terre des Sciences, Inra Angers, Institut national d'horticulture, Université d'Angers, Université catholique de l'Ouest, Groupe national interprofessionnel des semences et plants, CNFPT, OCIM, inspection académique de Maine-et-Loire, rectorat de Nantes, IUFM des Pays de la Loire, CDDP, Cité des sciences et de l'industrie, Musée botanique d'Angers, Jardin botanique de Nantes, Société d'horticulture d'Angers, Mission Val de Loire.
}

territorial), propriétaires de parcs et jardins, professionnels, artistes...

Il s'est ouvert sur une séance plénière riche en expériences. L'exemple de la Cité de la vigne et du vin, installée sur le domaine Inra de Pech Rouge (Aude), marque par son concept : un centre de culture scientifique expliquant au public comment s'élabore le vin, l'importance de la culture de la vigne et de l'obtention d'une baie de qualité, et par son originalité : jeter des ponts entre traditions viticoles et innovations apportées par la recherche (découvrir la recherche en marche). Hormis les aspects techniques et scientifiques, ce nouvel acteur culturel et touristique en Languedoc-Roussillon s'attache à intéresser le public à la conservation de la diversité végétale et à la préservation des paysages, avec notamment un parcours d'interprétation dans les vignes du domaine. De même, les missions des gestionnaires des parcs et jardins ont évolué en une vingtaine d'années au profit du public. Les jardins publics deviennent des lieux d'échanges et de médiations, comme les a présentés Jacques Soignon, directeur du service des Parcs et Jardins de la ville de Nantes. Les habitants participent au programme de réhabilitation des parcs publics traditionnels et des espaces d'habitat social; la conception en est totalement modifiée. Les jardins deviennent des lieux d'une grande mixité d'usage et sont partagés entre acteurs privés et publics. Cet enrichissement mutuel permet l'éclosion de nouvelles idées, les jardins à thème se multiplient et les nouvelles initiatives exploitent toutes les facettes du métier de jardinier, aussi bien celle de l'animation que celle de la communication.

Autre expérience présentée en séance plénière : le Conservatoire international des parcs et jardins et du 
paysage de Chaumont-sur-Loire. Jean-Paul Pigeat ${ }^{2}$ n'a pas manqué de souligner le caractère très expérimental du Festival des jardins de Chaumont, qui a nettement étonné le public dans les premiers temps. Néanmoins, le «transfert culturel» a eu lieu : on observe dès 1992 les premiers passages de l'expérimental au public, illustrés notamment à Blois par la création de ronds-points mêlant légumes et fleurs. Les guides et les jardiniers très présents au festival sont les médiateurs. Grâce à eux, le passage de l'expérimental au public peut se faire.

Parmi les différentes interventions des séances plénières, le programme transfrontalier France-Belgique est également à retenir. En effet, Régine Pattyn, directrice de l'association Chantier Nature (Lille), et Annick Marchal, chargée de mission à Espace Environnement (Charleroi), ont expliqué que les territoires du Nord-Pas-de-Calais et de la Wallonie présentent des problématiques similaires en matière de requalification d'espaces et d'appauvrissement de la biodiversité. Le traitement de ces questions passe notamment par des actions de proximité et une prise de conscience et de responsabilité de la part du particulier. Pour cela, le citoyen doit intégrer les dispositifs de coélaboration, voire de cogestion de projets. Ainsi, dans ce programme transfrontalier de promotion et de mise en œuvre d'aménagements verts participatifs, une méthodologie de mobilisation des habitants en vue d'une réappropriation du territoire a été élaborée. Ces premiers travaux communs ont permis de créer des outils d'interpellation et de sensibilisation, de mettre en place des évènements fédérateurs «symboliques »...

Autre temps fort de ce colloque, hormis les visites de sites de l'Anjou : les ateliers. Durant une matinée, quatre groupes se sont formés pour échanger sur les thèmes suivants : pratiques éducatives sur le temps scolaire; pratiques dans les jardins pour le grand public; développement touristique culturel et artistique autour du végétal; le végétal et l'insertion. Des exemples de pratiques de médiation ont été partagés au sein de chacun des ateliers. Pour le milieu scolaire, tous s'accordent à dire qu'il existe de réelles expériences : jardinage expérimental à l'école, visite active de jardins, concours d'écoles fleuries... Des partenariats entre professionnels, enseignants, animateurs de centre de culture scientifique technique et industrielle et chercheurs sont également tissés, afin de mener à bien ces pratiques.

L'ouverture des parcs et jardins au grand public s'inscrit également dans une démarche éducative. Là encore, les exemples sont nombreux: Parc oriental de Maulévrier (Maine-et-Loire), Conservatoire botanique de Brest, expérience originale avec le cours de botanique de la ville de Nantes. La dimension patrimoniale des jardins et du végétal a également été mise en valeur : patrimoine historique, culturel et biologique. Néanmoins, il faut faire

${ }^{2}$ J.-P. Pigeat est décédé depuis ce colloque. attention aux dérives commerciales, à l'amateurisme ou à la surfréquentation de certains parcs et jardins.

Lors de l'atelier « Développement touristique culturel et artistique autour du végétal», une multiplicité d'approches est ressortie des quatre projets présentés : les terrasses de l'évêché et les abords du château de Blois ; l'Harmas de Jean-Henri Fabre (Vaucluse) ; histoire et mémoire du végétal en Anjou ; expositions de l'artistebotaniste Liliana Motta. Chacun d'eux repose sur d'importantes recherches scientifiques, historiques et sociologiques. La dimension des publics et des médiations y est présente, mais en second lieu, découlant généralement de ces fondements. Chaque lieu appelle ses couleurs : poétique, scientifique, politique, touristique... et ses usages pour le public. Mais tous ces projets s'inscrivent dans un maillage remarquable de partenaires et de complices.

Dans le dernier atelier "Le végétal et l'insertion », le végétal s'est vraiment révélé comme le support idéal de la médiation, de l'insertion sociale. En effet, l'insertion par le jardin a un rôle social permettant de développer, notamment la solidarité, les liens communautaires, la créativité, la responsabilisation, la reconnaissance personnelle, la reconnaissance des autres. Les actions conduites aujourd'hui sont multiples, très diverses. Les partenariats sont forts et la mise en réseau, très présente. Il existe des réseaux comme celui du Jardin dans tous ses états, des Jardins de Cocagne, ou encore des associations d'insertion professionnelle telles que l'AAPIJ (Association pour l'action préventive et l'insertion de la jeunesse), des structures indépendantes, comme le Potager extraordinaire (Vendée).

Pour conclure ce colloque, le président du Muséum national d'histoire naturelle, Bernard Chevassusau-Louis, a proposé une visite du monde végétal selon quatre "saisons »: le monde végétal, fondement de l'aventure humaine; le monde végétal, un univers méconnu et incompris; le monde végétal, un perpétuel changement; le monde végétal et le défi des climats de demain. Ce voyage surprenant a éclairé le terme de biodiversité et a proposé quelques approches pour mieux l'appréhender. «La variation intraspécifique est une composante de la description de la biodiversité. Une autre est la diversité des associations d'espèces, c'est-à-dire les écosystèmes. Toutes les espèces ne sont pas toutes présentes au même endroit. Non seulement il faut décrire chaque espèce, mais aussi chacune des associations. Il ne suffit donc pas d'avoir la liste des espèces pour décrire la biodiversité. ». Cette conclusion a été comme un écho à l'intervention d'Aline Raynal-Roques, professeur honoraire du Muséum national d'histoire naturelle, et de Claude Figureau, du Jardin botanique de Nantes, «La biodiversité et la botanique aujourd'hui ». N'oublions pas la botanique, cette "science à part entière complexe et moderne, indispensable à l'exploitation de la puissance économique qu'est le végétal ». 
Le deuxième colloque national "Végétal et médiations culturelles » se tiendra les 26 et 27 octobre 2006 à Angers et aura pour thème : «Redécouvrir la nature ». La matinée du 27 sera composée de trois ateliers : «Pratiques éducatives à l'école, au collège, au lycée (général, professionnel et agricole) »; « Pratiques culturelles d'associations, de CCSTI, de CPIE, de PNR, de jardins nature et de musées »; " Pratiques d'aménageurs dans la ville, le quartier, le village et/ou l'espace rural ».

Les résumés des communications du colloque de 2004 sont disponibles sur demande auprès de Terre des Sciences, 5 rue Fleming, 49066 Angers cedex 01. Tél. : 02417214 21. Site Internet : www.terre-des-sciences.org

To access this journal online: www.edpsciences.org 